\title{
9q31.1q31.3 microdeletion syndrome
}

INSERM

\section{Source}

INSERM. (1999). Orphanet: an online rare disease and orphan drug data base.

9q31.1q31.3 microdeletion syndrome. ORPHA:401923

9q31.1q31.3 microdeletion syndrome is a rare, genetic, syndromic intellectual disability

characterized by mild intellectual disability, short stature with high body mass index, short neck with cervical gibbus and dysmorphic facial features. A metabolic syndrome, including type 2 diabetes, hypercholesterolemia and hypertension has also been reported. 